\title{
Volume-Time Index Is Not an Indicator of Outcome for Patients Undergoing CRS/HIPEC for Treatment of High-Grade Appendiceal Carcinomatosis
}

\author{
DANIEL M. DOTTA ${ }^{1 *}$, JOSHUA D. LANSOM ${ }^{1,2^{*}}$, OLIVER M. FISHER ${ }^{1,2,3}$, \\ NAYEF A. ALZAHRANI FRACS ${ }^{2,4}$, WINSTON LIAUW ${ }^{2,5}$ and DAVID L. MORRIS ${ }^{1,2}$ \\ ${ }^{1}$ St George \& Sutherland Clinical School, University of New South Wales, Sydney, Australia; \\ ${ }^{2}$ Liver \& Peritonectomy Unit, St George Hospital, Sydney, Australia; \\ ${ }^{3}$ School of Medicine, University of Notre Dame, Sydney, Australia; \\ ${ }^{4}$ College of Medicine, Al Imam Mohammad Ibn Saud Islamic University (IMSIU), Riyadh, Kingdom of Saudi Arabia; \\ ${ }^{5}$ Cancer Care Clinic, St George Hospital, Sydney, Australia
}

\begin{abstract}
Background/Aim: Completeness of cytoreduction score (CC-score) and tumour grade have been shown to be independent prognostic factors in patients undergoing cytoreductive surgery (CRS) and hyperthermic intraperitoneal chemotherapy (HIPEC) for the treatment of high-grade appendiceal carcinomatosis (PMCA). In patients with colorectal cancer with peritoneal metastases (CRPM) that undergo CRS/HIPEC, volume time index (VTI) has been shown to be an independent prognostic factor for overall survival (OS). This analysis was conducted to evaluate VTI as a prognostic factor in PMCA. Patients and Methods: We performed A retrospective cohort study of 131 patients who underwent CRS-HIPEC for PMCA from 1996 to 2017. VTI was calculated and analyses were performed to determine the association with patients' outcomes. Results: Median overall survival by high vs. low VTI groups were 69 months (95\%CI=45-NA) vs. 63 months [95\% Confidence Interval $(C I)=48$-not available (NA)], with an associated difference in 5 -year survival rates of $52.1(95 \% C I=39.5-68.8)$ vs. 50.6 (95\% CI=35.2-72.6) respectively, with a non-significant $p$ value of 0.968. For the 82 patients with complete recurrence data, the median RFS stratified by high vs. low VTI were 16
\end{abstract}

*These Authors contributed equally as co-first authors.

Correspondence to: David L. Morris, Hepatobiliary and Surgical Oncology Unit, Department of Surgery, St George Hospital, University of New South Wales, Research \& Education Centre (410 South St), Kogarah, Sydney, NSW 2217, Australia. Tel: +61 291132590, Fax: +61 291133997, e-mail: david.morris@unsw.edu.au

Key Words: High-grade appendiceal carcinomatosis, peritoneal metastases, cytoreductive surgery, intraperitoneal chemotherapy, volume-time-index. months (95\%CI=10-25) vs. 20 months (95\%CI=13-34) respectively, with no statistically significant difference in 5 year recurrence-free survival (RFS). Conclusion: VTI for PMCA was not shown to be correlated with overall survival (OS) for patients undergoing CRS/HIPEC, and suggests that the rate of tumour growth does not affect the patients' outcome. Neither high PCI nor rapid tumour growth following primary tumour resection should, therefore, be a contraindication for CRS/HIPEC in patients with PMCA.

Malignant neoplasms of the appendix are extremely rare, with an incidence rate that has been estimated to be approximately 0.12 per $1,000,000$ people (1). Appendiceal cancer accounts for $0.4 \%$ of all gastrointestinal tumours, with a 5-year survival rate of $6 \%$ for untreated high-grade appendiceal carcinomatosis (PMCA) $(2,3)$. PMCA is also a synonymous term, and refers to: i) pseudomyxoma peritonei (PMP) with high-grade haematological features, ii) high grade mucinous carcinoma peritonei, and iii) peritoneal mucinous carcinomatosis (4).

Since the implementation of cytoreductive surgery and hyperthermic intraperitoneal chemotherapy (CRS/HIPEC) as the primary modality of treatment over the last two decades, 5 -year survival rates have increased to $59 \%$ for patients with PMCA, when a completeness of cytoreduction score (CCscore) of 0 or 1 is achieved (3). Current prognostic factors for patients undergoing CRS/HIPEC are: i) histological subtype of appendiceal neoplasms, ii) peritoneal cancer Index (PCI), and iii) CC-score (4).

Volume-time-index (VTI) is the ratio between peritoneal cancer index (PCI) pre-intervention, and the number of months since the primary tumour resection. A recent retrospective study identified VTI of colorectal cancer with peritoneal metastases (CRPM) as being a significant 
Table I. Comparison of clinicopathologic and demographic features of patients with PMCA undergoing CRS/HIPEC with low vs high VTI.

\begin{tabular}{|c|c|c|c|c|}
\hline & $\begin{array}{c}\text { All patients } \\
\mathrm{n}=131\end{array}$ & $\begin{array}{l}\text { Patients with a low VTI } \\
n=50\end{array}$ & $\begin{array}{l}\text { Patients with a high VTI } \\
n=81\end{array}$ & $p$-Value* \\
\hline Age $(\text { years })^{* *}$ & $55(45-65)$ & $59(44-65)$ & $53(45-64)$ & 0.45 \\
\hline Gender, n (\%) & & & & 0.32 \\
\hline Male & $48(36.7)$ & $21(42.0)$ & $27(33.3)$ & \\
\hline Female & $83(63.3)$ & $29(58.0)$ & $54(66.7)$ & \\
\hline ASA Score, $\mathrm{n}(\%)$ & & & & 0.68 \\
\hline ASA I-II & $39(29.8)$ & $16(33.0)$ & $23(28.4)$ & \\
\hline ASA III-IV & $86(65.6)$ & $32(64.0)$ & $54(66.7)$ & \\
\hline Missing data & $6(4.6)$ & $2(4.0)$ & $4(4.9)$ & \\
\hline ECOG performance status, $\mathrm{n}(\%)$ & & & & 0.79 \\
\hline $0-1$ & $114(87.0)$ & $43(86.0)$ & $71(87.7)$ & \\
\hline $2-3$ & $17(13.0)$ & $7(14.0)$ & $10(12.3)$ & \\
\hline Preoperative weight $(\mathrm{kg})$ & $71(61-83)$ & $71(62-86)$ & $70(61-82)$ & 0.71 \\
\hline Preoperative albumin levels, $\mathrm{n}$ (range) & $38(35-40)$ & $38(36-41)$ & $39(36-40)$ & 0.32 \\
\hline Pre-CRS CEA level, n (range) & $5(2-19)$ & $7(320)$ & $5(2-18)$ & 0.30 \\
\hline Pre-CRS CA19.9 level, n (range) & $20(9-82)$ & $24(8-110)$ & $18(9-74)$ & 0.73 \\
\hline Signet ring cell histology, $\mathrm{n}(\%)$ & & & & 0.16 \\
\hline No & $96(73.3)$ & $33(66.0)$ & $63(77.8)$ & \\
\hline Yes & $35(26.7)$ & $17(34.0)$ & $18(22.2)$ & \\
\hline Time from primary CRC to CRS (months), $\mathrm{n}$ (range) & $10(4-28)$ & $36(16-51)$ & $5(3-14)$ & $<0.001$ \\
\hline Peritoneal carcinomatosis index $(\mathrm{PCI}), \mathrm{n}$ (range) & $20(8-30)$ & $18(5-30)$ & $21(12-30)$ & $<0.001$ \\
\hline Completeness of Cytoreduction (CC) Score, $\mathrm{n} \%$ ) & & & & 0.24 \\
\hline 0 & $91(69.5)$ & $38(76.0)$ & $53(65.4)$ & \\
\hline 1 & $40(30.5)$ & $12(24.0)$ & $28(34.6)$ & \\
\hline Operating time, $\mathrm{h}$ (range) & $9(6.4-10.6)$ & $7(6.4-10.6)$ & $9(6.9-11.0)$ & 0.46 \\
\hline Blood transfusion, units (range) & $4(0.5-7.5)$ & $4(0.0-7.0)$ & $5(2.0-8.0)$ & 0.34 \\
\hline Intensive care unit length of stay, days (range) & $2(1.0-4.0)$ & $2(1.2-3.7)$ & $2(1.0-4.0)$ & 0.92 \\
\hline High-dependency unit length of stay, days (range) & $2(0.0-5.0)$ & $2(1.0-5.0)$ & $2(0.0-5.0)$ & 0.86 \\
\hline Total hospital length of stay, days (range) & $22(13.0-27.0)$ & $22(12.3-32.3)$ & $22(14.0-39.0)$ & 0.41 \\
\hline Complication grade, $\mathrm{n}(\%)$ & & & & 0.77 \\
\hline Grade 0 & $21(16.0)$ & $11(22.0)$ & $10(12.3)$ & \\
\hline Grade 1 & $6(4.6)$ & $2(4.0)$ & $4(4.9)$ & \\
\hline Grade 2 & $40(30.5)$ & $13(26.0)$ & $27(33.4)$ & \\
\hline Grade 3 & $32(24.4)$ & $12(24.0)$ & $20(24.7)$ & \\
\hline Grade 4 & $28(21.4)$ & $11(22.0)$ & $17(21.0)$ & \\
\hline Grade 5 & $4(3.1)$ & $1(2.0)$ & $3(3.7)$ & \\
\hline In-hospital death, n (\%) & $4(3.1)$ & $1(0.8)$ & $3(2.3)$ & - \\
\hline Number of deaths during follow-up, n (\%) & $48(36.6)$ & $19(14.5)$ & $29(22.1)$ & 0.95 \\
\hline Recurrence, $\mathrm{n}(\%)$ & $71(54.2)$ & $22(16.8)$ & $49(37.4)$ & 0.09 \\
\hline 5 -year survival rate $(\%)$ & 53.1 & 50.6 & 52.2 & - \\
\hline
\end{tabular}

* $p$-Values were calculated with Pearson's chi-squared or Fisher's exact test comparing the low $v s$. high group where appropriate. **All continuous variables are presented as median with interquartile range (IQR) in brackets unless denoted otherwise. PMCA: High-grade appendiceal carcinomatosis; CRS: cytoreductive surgery; HIPEC: hyperthermic intraperitoneal chemotherapy; VTI: volume-time index; ASA: physical status classification system; ECOG: Eastern Cooperative Performance Group; CEA: carcinoembryonic antigen; CA 19.9: cancer antigen 19.9.

prognostic factor (5). Our study therefore aimed to evaluate the prognostic value of VTI in PMCA.

\section{Patients and Methods}

Treatment setting and patient selection. Data from all patients with PMCA managed at St George Hospital (Sydney, Australia) from February 1996 to June 2017 with CRS and HIPEC were collected in a prospectively maintained peritoneal surface oncology database, which included all perioperative and operative clinicopathological and tumour-related variables.

Patients included had the following criteria: i) undergoing their first CRS/HIPEC, ii) no extra-abdominal disease, iii) no liver metastases, iv) a $\mathrm{PCI} \geq 1$, and v) a CC-score of 0 or $1 \quad(n=131)$. Patients with CC-scores $\geq 2$ and unable to undergo CRS/HIPEC were excluded from the analysis, as were patients that underwent simultaneous primary tumour resection and CRS/HIPEC. All patients had previously consented to the collection, storage, and use of their data, for the purposes of scientific analysis. 
CRS and HIPEC. A mid-line laparotomy was performed and PCI was calculated $(6,7)$. CRS was then performed as described by Sugarbaker et al. (7). Post CRS, the CC-score was calculated and HIPEC was administered (8).

Statistical analysis. Continuous variables were compared using Student's $t$-, Wilcoxon rank-sum, one-way analysis of variance ANOVA and/or Kruskal-Wallis tests, as appropriate. Normal distribution was achieved using log-transformation data as required. Pearson's $\chi^{2}$ or Fisher's exact test were used to compare differences between proportions of categorical data. Data are reported as median values with inter-quartile range (IQR) unless denoted otherwise.

Individual patient VTI was calculated by dividing the tumour volume, expressed as PCI, by the time from primary tumour resection to the date of CRS/HIPEC (in months). Once the VTI was generated, cohort stratification occurred at the ratio of 1 to assess the prognostic impact of patients having a high (ratio $\geq 1$ ) or low (ratio <1) VTI. The cut-off of 1 was chosen to facilitate the reproducibility and future validation of the VTI calculation by choosing a simple ratio that equates to "one PCI per month".

The impact of the VTI on patients' short-and long-term survival outcomes were assessed post stratification. Stratified overall (OS) and recurrence-free (RFS) survival times were plotted using the Kaplan-Meier method and were compared using log-rank test.

Cohort data were analysed in order to assess the survival impact of time from the primary resection to CRS/HIPEC as an independent variable, by stratifying patients' outcomes every6 months. Uni- and multi-variable Cox regression analysis was performed to assess the independent prognostic value of the VTI for both RFS and OS. Overall survival was defined as the time from CRS to date of last follow-up with death from any cause, whereas recurrence free survival was defined as the time from CRS to recurrence at any site. All $p$-Values $<0.05$ were regarded as statistically significant.

\section{Results}

Patient demographics. Median patient age was 55 years [Interquartile range $(\mathrm{IQR})=44.5-64.5]$, with a female majority $(n=83,63.4 \%)$. The median time from the resection of the primary tumour to CRS/HIPEC was 10.3 months (IQR=4-28). Median PCI of the population was 20 (IQR=8$30)$. Median operating time was 9 hours $(\mathrm{IQR}=6.5-10.7)$. The median length of hospital stay was 22 days (IQR=13.0-37.0), and a total of 4 deaths in hospital. Baseline demographics, clinical and pathological features are outlined in Table I.

During the follow-up period there were 48 deaths (36.6\%), $19(14.5 \%)$ with low VTI and $29(22.1 \%)$ with high VTI. The overall cohort median survival time was 63 months [95\% confidence interval $(\mathrm{CI})]$.

This translated to a 5-year survival rate of $51.3 \%$ (95\% CI=41.1-64.1).

Patients with a low VTI $(<1)$ were noted to have longer time frames between initial resection and CRS/HIPEC (35.9 months, IQR=16.0-51.3 vs. 5.1 months, IQR=2.9-13.5, $p<0.001)$. No further statistically significant difference between high and low VTI groups was evident.

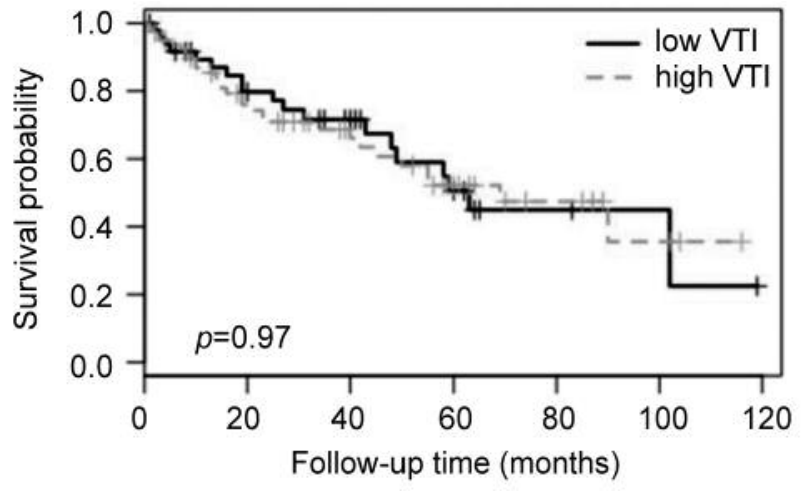

Figure 1. Overall survival based on volume-time index (VTI).

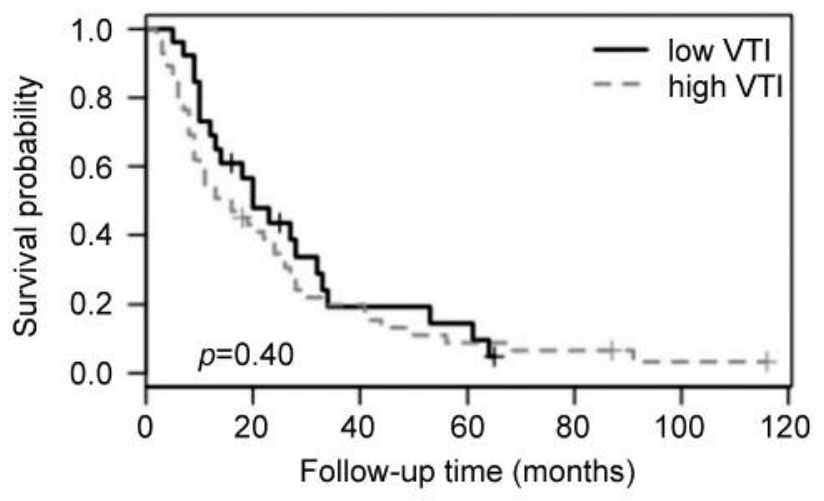

Figure 2. Recurrence-free survival based on volume-time index (VTI).

Impact of volume-time index on patient overall and recurrence free survival. The impact of VTI on OS is shown in Figure 1 and Table II. The median overall survival by high vs. low VTI groups were 69 months $(95 \% \mathrm{CI}=45-\mathrm{NA})$ vs. 63 months $(95 \% \mathrm{CI}=48-\mathrm{NA})$, with an associated difference in 5year survival rates of $52.1(95 \% \mathrm{CI}=39.5-68.8) v s .50 .6$ $(95 \% \mathrm{CI}=35.2-72.6)$ respectively, with a non-significant $p$ Value of 0.968 .

Patient recurrence free survival and its VTI impact is shown in Figure 2 and Table III. Analysis was not complete due to 49 observations missing. From the 82 remaining, the median RFS stratified by high $v s$. low VTI were 16 months (95\%CI=10-25) vs. 20 months (95\%CI=13-34), respectively. Overall 5-year RFS was $14.5 \%$ of low VTI $(95 \% \mathrm{CI}=5.1$ 40.8) vs. $8.8 \%$ of high VTI $(95 \% \mathrm{CI}=3.5-22.2)$ patients, with a non-significant $p$-Value of 0.396 .

Cox regression analysis for overall and recurrence free survival. A uni- and multi-variable Cox regression model, adjusting for survival influencing confounding factors, was 
Table II. Analysis of overall survival of patients with PMCA undergoing CRS/HIPEC stratified by VTI.

\begin{tabular}{lcccc}
\hline & $\mathrm{VTI}<1(\mathrm{n}=50)$ & $95 \% \mathrm{CI}$ & $\mathrm{VTI}>1(\mathrm{n}=81)$ & $95 \% \mathrm{CI}$ \\
\hline $\begin{array}{l}\text { Median overall survival (months) } \\
\text { Median survival (\%) }\end{array}$ & 63 & $48-\mathrm{NA}$ & 69 & \\
1 year & & & & \\
3 years & 89.3 & $80.9-98.6$ & 85.4 & $77.7-93.8$ \\
5 years & 71.6 & $59.1-86.8$ & 68.6 & $58.1-81.1$ \\
\end{tabular}

PMCA: High-grade appendiceal carcinomatosis; CRS: cytoreductive surgery; HIPEC: hyperthermic intraperitoneal chemotherapy; VTI: volumetime index.

Table III. Analysis of recurrence free survival in patients with PMCA undergoing CRS/HIPEC stratified by VTI.

\begin{tabular}{lcccc}
\hline & VTI $<1(\mathrm{n}=26)$ & $95 \% \mathrm{CI}$ & $\mathrm{VTI}>1(\mathrm{n}=56)$ & $95 \% \mathrm{CI}$ \\
\hline $\begin{array}{l}\text { Median recurrence free survival (months) } \\
\text { Recurrence free survival (\%) }\end{array}$ & 20 & $13-34$ & 16 & $10-25$ \\
1 year & 69.0 & $53.3-89.4$ & 54.4 & $42.7-69.4$ \\
3 years & 19.3 & $8.90-45.7$ & 19.8 & $11.3-34.9$ \\
5 years & 14.5 & $5.20-40.6$ & 8.80 & $3.50-22.2$ \\
\hline
\end{tabular}

PMCA: High-grade appendiceal carcinomatosis; CRS: cytoreductive surgery; HIPEC: hyperthermic intraperitoneal chemotherapy; VTI: volumetime index.

also conducted. This found that ECOG 2-3 [adjusted hazard ratio $(\mathrm{HR})=2.73,95 \% \mathrm{CI}=1.39-5.38, p=0.004]$, Signet cell morphology (adjusted $\mathrm{HR}=2.08,95 \% \mathrm{CI}=1.15-3.77, p=0.005$ ), Clavien-Dindo score (adjusted $\mathrm{HR}=2.08,95 \% \mathrm{CI}=1.15-3.77$ ), $p=0.01$ ), and PCI (adjusted $\mathrm{HR}=3.13$, 95\% $\mathrm{CI}=1.33-7.38$, $p=0.009)$ were all independently associated with a poorer OS. However, there was no demonstrated correlation between measured variables and recurrence free survival rates.

On the multi-variable Cox regression analysis, no significant association between high VTI and poorer RFS was identified (adjusted $\mathrm{HR}=1.36,95 \% \mathrm{CI}=0.77-2.40, p=0.28$ ).

\section{Discussion}

The aim of this study was to examine if the rate of peritoneal tumour growth, measured using VTI, is associated with worse OS and RFS in patients with PMCA. High VTI has been previously shown to have a negative impact on OS and RFS in patients with CRPM undergoing CRS/HIPEC (5). This study demonstrated a median overall survival of 23 months vs. 44 months in patients with high and low VTI respectively, with a difference in 5-year OS of $20.3 \%$ vs. $40.1 \%$ (5). Our study showed that a higher rate of peritoneal tumour growth was not associated with a decrease in OS or RFS.

Notably, patients with a low VTI had longer time frames between initial tumour resection and CRS/HIPEC with a median OS of 35.9 months vs. 5.1 months for low $v s$. high VTI, respectively. A possible explanation for this could be that PMCA with lower growth rates did not manifest symptoms that would have expedited treatment for those patients; however, this cannot be definitively concluded.

Overall, the evidence provided in this study determined that there is no statistically significant difference in OS or RFS relative to tumour growth rate, and that rapid tumour growth rate and/or high PCI are not contraindications for CRS/HIPEC therapy in PMCA patients. This is likely due to the distinct genetic differences between PMCA and colorectal cancer, with separate genes attributed to behaviour of tumour growth and dissemination (9). In particular, trefoil factors 1 and 2 have been linked to tumour genesis in gastrointestinal cancers, while MUC-2 and MUC-5AC are directly linked to the physiochemical properties of mucin formation, which result in peritoneal surface disease. Gene profiling analysis has confirmed reduced expression of these genes in colorectal cancers compared to PMCA (9).

Current guidelines for the treatment of PMCA advise that a PCI $>20$ is not a contraindication for CRS/HIPEC, provided there is no evidence of extra-abdominal disease, extraperitoneal disease, or an unknown primary tumour (10). Our results support these guidelines, with calculated 5-year survival rates of $50.6 \%$ (median=63 months) and $52.1 \%$ (median=69 months) in low vs. high VTI groups, respectively. Given the significant variations in OS between 
PMCA and CRPM and the known genetic variations, a potential next step would be to analyse VTI against genetic profiles in order to identify possible correlations between tumour growth rate and known tumour genes.

Unlike the Colorectal Peritoneal Score (COREP) (11), a prognostic scoring system for appendiceal cancers does not currently exist. The current known prognostic indicators of increased OS and RFS post CRS/HIPEC are lower value of i) $\mathrm{CC}$ and ii) PCI scores, but they will still be dependent on the tumour subtype pathology (12).

This study is not without its limitations, starting with a selection bias for all patients who underwent CRS/HIPEC, due to the nature of retrospective cohort studies. Additionally, patients who underwent simultaneous primary tumour resection and CRS/HIPEC were also excluded, thus limiting the ability to generalise our findings. Finally, the overall number of patients is quite small, likely as a result of the low incidence rate of PMCA; however, despite this small number there was no evident trend of increased survival in one group over the other.

Ongoing analysis of future cases is, thus, essential for the development of better prognostic tools.

In conclusion, no difference in PMC patients' outcome was found between slow growing $(\mathrm{VTI}<1)$ or fast growing (VTI $>1$ ) PMCA, and as such VTI was not shown to provide any further prognostic predictions for patients undergoing CRS/HIPEC. Neither high PCI nor rapid tumour growth following primary tumour resection, should therefore be a contraindication for CRS/HIPEC in patients with PMCA, as per current treatment guidelines.

\section{Conflicts of Interest}

None to declare.

\section{Authors' Contributions}

All Authors participated in drafting and revising the article content and approved the final version of the manuscript prior to submission.

\section{References}

1 Xie X, Zhou Z, Song Y, Wenhan L, Dongmei D, Chengxue D and Hao Z: The management and prognostic prediction of adenocarcinoma of appendix. Sci Rep 6: 39027, 2016. PMID: 27982068. DOI: 10.1038/srep39027

2 Ruoff C, Hanna L, Zhi W, Shahzad G, Gotlieb V and Saif MW: Cancers of the appendix: review of the literatures. ISRN Oncol 2011: 728579, 2011. DOI:10.5402/2011/728579.

3 Huang Y, Alzahrani NA, Chua TC and Morris DL: Histological subtype remains a significant prognostic factor for survival outcomes in patients with appendiceal mucinous neoplasm with peritoneal dissemination. Dis Colon Rectum 60: 360-367, 2017 PMID: 28267002. DOI: 10.1097/DCR.0000000000000719
4 Carr NJ, Cecil TD, Mohamed F, Sobin LH, Sugarbaker PH, Gonzalez-Moreno S, Taflampas P, Chapman and Moran BJ: A consensus for classification and pathologic reporting of pseudomyxoma peritonei and associated appendiceal neoplasia: The results of the Peritoneal Surface Oncology Group International (PSOGI) modified Delphi process. Am J Surg Pathol 40: 14-26, 2016. PMID: 26492181. DOI: 10.1097/PAS.0000000000000535

5 Kozman M, Fisher OM, Valle SJ, Alzahrani N, Liauw W and Morris DL: The volume-time index (VTI) is prognostic in patients with colorectal cancer peritoneal metastases undergoing cytoreductive surgery and intraperitoneal chemotherapy. Am J Surg pii: S0002-9610(19)30158-8, 2019. PMID: 30982572. DOI: 10.1016/j.amjsurg.2019.03.023

6 Jacquet PS: Current methodologies for clinical assessment of patients with peritoneal carcinomatosis. J Exp Clin Cancer Res 15: 49-58, 1996.

7 Sugarbaker PH and Ryan DP: Cytoreductive surgery plus hyperthermic perioperative chemotherapy to treat peritoneal metastases from colorectal cancer: standard of care or an experimental approach? Lancet Oncol 13(8): 362-369, 2012. PMID: 22846841. DOI: 10.1016/S1470-2045(12)70210-3

8 Chua TC, Liauw W, Zhao J and Morris DL: Comparative analysis of perioperative intraperitoneal chemotherapy regimen in appendiceal and colorectal peritoneal carcinomatosis. Int $\mathbf{J}$ Clin Oncol 18(3): 439-446, 2013. PMID: 22415741. DOI: 10.1007/s10147-012-0397-5

9 Levine EA, Blazer III DG, Kim MK, Shen K, Stewart JH, Guy $\mathrm{C}$ and Hsu DS: Gene expression profiling of peritoneal metastases from appendiceal and colon cancer demonstrates unique biologic signatures and predicts patient outcomes. J Am Coll Surg 214(4): 599-606, 2012. PMID: 22342786. DOI: 10.1016/j.jamcollsurg.2011.12.028

10 Dubé P, Sideris L, Law C, Mack L, Hasse E, Giacomantonio C, Govindarajan A, Krzyzanowska MK, Major P, McConnell Y, Temple W, Younan R and McCart JA: Guidelines on the use of cytoreductive surgery and hyperthermic intraperitoneal chemotherapy in patients with peritoneal surface malignancy arising from colorectal or appendiceal neoplasms. Curr Oncology 22(2): 100-112, 2015. PMID: 25908915. DOI: 10.3747/co.22.2058

11 Cashin PH, Graf W, Nygren P and Mahteme H: Patient selection for cytoreductive surgery in colorectal peritoneal carcinomatosis using serum tumor markers: an observational cohort study. Ann Surg 256(6): 1078-1083, 2012. PMID: 22580940. DOI: 10.1097/SLA.0b013e318254f 281

12 Loftus TJ, Raymond SL, Sarosi GA, Croft CA, Smith RS, Efron PA, Moore FA, Brakenridge SC, Mohr AM and Jordan JR: Predicting appendiceal tumors among patients with appendicitis. J Trauma Acute Care Surg 82(4): 771-775, 2017. PMID: 28099380. DOI: 10.1097/TA.0000000000001378
Received November 5, 2019

Revised November 17, 2019

Accepted November 19, 2019 